\title{
NOTIZIE SUI COLLABORATORI DI QUESTO NUMERO
}

Giacomo Sani, Professor Emeritus di Scienza politica presso la Ohio State University, insegna alla facoltà di Scienze politiche di Pavia. Tra le sue opere più recenti Il mercato elettorale (1986, con $\mathrm{R}$. Mannheimer).

CARLO FUSARO è ricercatore confermato in diritto pubblico e incaricato di Storia delle istituzioni politiche nella Facoltà di Scienze politiche «Cesare Alfieri» di Firenze.

Paolo Segatti è ricercatore presso il dipartimento di Studi politici e sociali dell'Università di Pavia.

ALDO DI VIRGILIO è ricercatore di Scienza politica presso l'Università della Calabria. È autore di diversi saggi sui sistemi elettorali e sui sistemi partitici.

Liborio MatTina insegna Scienza della politica nella Facoltà di Scienze politiche di Trieste. Tra i suoi lavori ricordiamo Gli industriali e la democrazia (1991).

LUCA RICOLFI insegna Metodologia delle scienze sociali nell'Università di Torino. Fra i suoi ultimi lavori: Tre variabili. Un'introduzione all'analisi multivariata (1993) e L'ultimo parlamento (1993).

Stefano BARTOLini insegna Scienza della politica all'Università di Ginevra. Tra i suoi lavori più recenti: Identity and competition (con P. Mair 1990) e On time and comparative researcb (1993). 
ROBERTo D'Alimonte insegna Sistema politico italiano all'Università di Firenze. Ha pubblicato vari saggi sulla teoria delle scelte razionali, sul sistema partitico italiano e sul rapporto tra democrazia e competizione elettorale.

Alessandro Chiaramonte è dottorando in Scienza della politica. La sua tesi di laurea sul sistema elettorale italiano ha ottenuto nel 1993 il Premio Celso Ghini della Società italiana di Studi elettorali.

LuCA Verzichelli è dottorando in Scienza della politica all'Università di Firenze. È autore di alcuni articoli sulla classe politica italiana e sui parlamenti europei. 